\title{
An ambulatory dyskinesia monitor
}

\author{
A J Manson, P Brown, J D O’Sullivan, P Asselman, D Buckwell, A J Lees
}

National Hospital for Neurology and Neurosurgery, Queen Square, London, UK A J Manson P Brown

J D O'Sullivan

A J Lees

The Middlesex Hospital, London, UK A J Manson

J D O'Sullivan

P Asselman

D Buckwell

A J Lees

MRC Human

Movement and

Balance Unit, Queen

Square, London, UK

P Brown

Correspondence to:

Dr A J Lees, National

Hospital for Neurology and

Neurosurgery, Queen

Square, London

WC1N 3BG, UK

email a.lees@ion.ucl.ac.uk

Received 22 March 1999 and in revised form

12 July 1999

Accepted 7 September 1999

\begin{abstract}
Objectives-New treatments are now becoming available for the management of levodopa induced dyskinesias in Parkinsons's disease. However, assessment of their efficacy is limited by the inadequacies of current methods of dyskinesia measurement. The objective was to develop and validate a portable device capable of objectively measuring dyskinesias during normal daily activities.

Methods-A portable device was developed based on a triaxial accelerometer, worn on the shoulder, and a data recorder that can record levodopa induced dyskinesias. A computer program plots raw acceleration and acceleration over $0.5 \mathrm{~Hz}$ frequency bands against time. The acceleration in the different bands can then be compared with the raw acceleration trace, enabling identification and exclusion of confounding activities such as tremor and walking, which have a characteristic appearance on the trace. The validity of this device was assessed on 12 patients and eight age matched controls by comparing accelerations in the 1-3 $\mathrm{Hz}$ frequency band with established clinical dyskinesia rating scales. While wearing the monitor, subjects were videorecorded sitting and during dyskinesia provocation tasks, including mental activation tasks, eating, drinking, writing, putting on a coat, and walking. The dyskinesias were graded with both modified abnormal involuntary movement (AIM) and Goetz scales. The clinical ratings were then compared with the mean acceleration scores.

Results-Acceleration in the 1-3 Hz frequency band correlated well against both scales, during all individual tasks. Acceleration produced by normal voluntary activity (with the exception of walking, which produced large accelerations, even in controls) was small compared with dyskinetic activity. With walking excluded, the mean acceleration over the rest of the recording time correlated strongly with both the modified AIM (Spearman's rank $(r=0.972, p<0.001)$ and Goetz $(r=0.951$, $\mathrm{p}<0.001)$ scales.
\end{abstract}

Conclusions-This method provides an accurate, objective means for dyskinesia assessment, and compares favourably with established methods currently used. (F Neurol Neurosurg Psychiatry 2000;68:196-201)

Keywords: levodopa induced dyskinesias; ambulatory monitoring; accelerometers
Disabling and distressing levodopa induced dyskinesias (LID) represent an important therapeutic challenge in the later stages of Parkinson's disease, occurring in most patients after 5-10 years of treatment. Reduction of levodopa dosage may improve them but nearly always at the expense of increasing parkinsonian disability. New pharmacological and surgical treatments for this problem are now under evaluation. ${ }^{12}$ However, the highly variable nature and complexity of dyskinesias means that standard methods of assessment with semiquantitative rating scales may fail to show improvement. Patients often notice that their dyskinesias are at their worst during everyday activities, such as eating or using the telephone, and may build up towards the later part of the day. ${ }^{3}$ It is often difficult to recreate these situations in a clinical or laboratory setting to enable more accurate, objective rating of the dyskinesias.

Video recorded dopaminergic challenges are commonly used in an attempt to capture these movements, but have several limitations. However rigorously standardised, there may still be significant intrapatient variation, due to uncontrollable factors, such as emotional stress or quality of sleep. The chosen activation tasks may not induce dyskinesias in some patients, and occasionally patients may become less dyskinetic or even freeze when aware of being filmed. Furthermore, accurate video rating is highly demanding on clinical time, requiring two clinicians to view each segment of recording (often repeatedly when topographical grading is required). The commonly used clinical rating scales also have shortcomings. The abnormal involuntary movement scale (AIMS) ${ }^{4}$ was originally developed for assessment of tardive dyskinesia, and thus has an emphasis on orofacial dyskinesias. It includes topographical assessment of movement, but has no descriptive anchors to aid grading. The Goetz scale $^{5}$ (also referred to as the modified Obeso or Rush scale) does incorporate helpful descriptive anchors and makes some distinction between dyskinesia types. However, it is primarily designed to look at secondary disability, rather than the amount of excess movement, and has no topographical categories.

Patient diaries form an important part of dyskinesia assessment but, although helpful, rely on patient awareness and compliance, and do not measure important outcomes such as secondary effects of dyskinesia on quality of life. Although many patients are able to successfully learn self rating of disease features, ${ }^{6}$ the validity of such unsupervised self assessments can be extremely variable. ${ }^{7}$ Thus some form of simple portable device, which 
Table 1 Description of tasks during recording:

\begin{tabular}{|c|c|c|}
\hline $\begin{array}{l}\text { Task } \\
\text { No }\end{array}$ & Description & $\begin{array}{l}\text { Time spent } \\
\text { performing } \\
\text { task }\end{array}$ \\
\hline \multicolumn{3}{|c|}{ "Sitting tasks": } \\
\hline 1 & Still, at rest, in silence & 1 minute \\
\hline 2 & Mental taxation (mental arithmetic or mini mental state exam questions) & 1 minute \\
\hline 3 & Conversation & 1 minute \\
\hline \multicolumn{3}{|c|}{ Motor tasks: } \\
\hline 4 & Writing & 1 minute \\
\hline 5 & Drinking & 1 minute† \\
\hline 6 & Preparing toast (cutting toast and spreading with butter and jam) & 1 minute \\
\hline 7 & Eating toast & 1 minute \\
\hline 8 & Putting on and buttoning a coat & 1 minuteł \\
\hline 9 & Walking & 1 minute \\
\hline
\end{tabular}

${ }^{\star}$ Monitoring continued during the periods of relaxation between tasks.

†Periods of drinking were summed to total about 1 minute.

$\ddagger$ Patients took varying times to put on the coat, but averaged about one minute.

All patients were left to finish the task, where possible.

can record dyskinesias over a long period of time, would be extremely useful.

Complex equipment such as motion analysis camera systems and polymyography can provide objective data but only for a limited period and in a laboratory setting. In the past, accelerometers have been used to try and quantify these involuntary movements but have been limited by the inability to differentiate between dyskinesias, tremor, and volitional movement. ${ }^{8-10}$ Our objective was to develop and validate a practical, portable device, which could provide a more descriptive record of movements to avoid these disadvantages. (An abstract of the preliminary study has already been published. ${ }^{11}$ )

\section{Methods}

THE AMBULATORY MONITOR

The ambulatory device consisted of three orthogonally mounted miniature uniaxial piezoresistive accelerometers (EGAS-FS25,Entran 1td, Watford, UK). Each had linear sensitivity $( \pm 0.5 \mathrm{~dB})$ up to $4 \mathrm{kHz}$. The mounting block containing the accelerometers was

A

Vertical

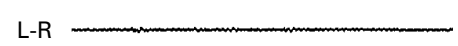

AP

C

Vertical

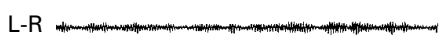

B

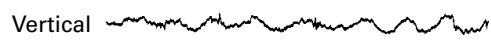

AP
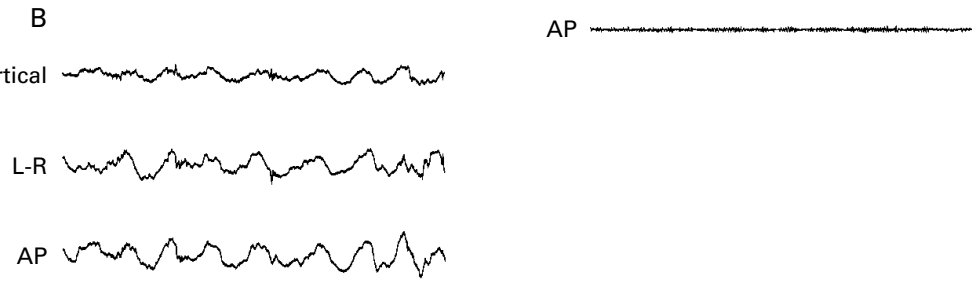

E

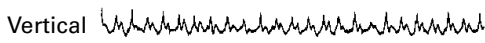

taped to the patient's shoulder using Micropore adhesive surgical tape and then connected to a low noise purpose built portable amplifier driven by a rechargable (nickel metal hydride) battery pack. The battery pack had a life of 15 hours during usage. Acceleration signals were first filtered $0-32 \mathrm{~Hz}$, using a steep CR type filter ( $40 \mathrm{~dB} /$ decade). The signal was then sampled at $64 \mathrm{~Hz}$ and collected on a portable data recorder (modified MS40 data recorder, Micromed Electronics Ltd, Woking, UK). This was powered by an internal (PP3) battery, which needed to be replaced after 24 hours of use. The amplifier, battery pack, and data recorder were worn attached to a belt around the waist and weighed $1.5 \mathrm{~kg}$ in total. At the end of a recording session the acceleration signals were downloaded to a portable PC. Custom written software divided data into blocks of equal length (128 data points) on which a finite Fourier transform was performed. The results (acceleration amplitude) from each 4 second period (two blocks) were averaged. These points were plotted against time, separately for $190.5 \mathrm{~Hz}$ steps from $0.5-10 \mathrm{~Hz}$. Acceleration in the three planes could be plotted either separately or as an arithmetic sum. The raw acceleration signal and acceleration in the 1-3 $\mathrm{Hz}$ bands alone could also be plotted. Total acceleration, either raw or in selected frequency bands, could be measured between two screen cursors, and was divided by the time between the cursors in seconds to give total acceleration per second. Raw acceleration was expressed in $\mathrm{g}$ and the device calibrated by turning each of the three uniaxial accelerometers on their side, to give $1 \mathrm{~g}$ output in the three orthogonal directions. Acceleration in different frequency bands was expressed in arbitrary units.

D

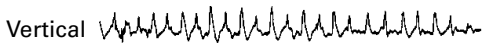

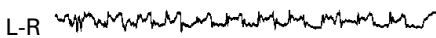

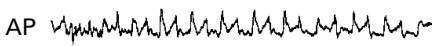

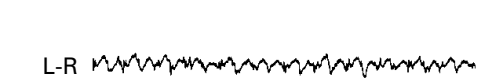

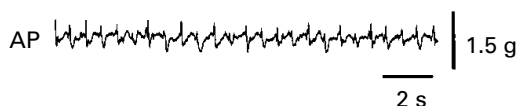

Figure 1 Traces from the raw data record: $(A$ and $B)$ voluntary movement (writing) in $(A)$ control, compared with (B) dyskinetic patient. (C) Rest tremor in a patient when off. ( $D$ and $E$ ) The characteristic appearance of walking, in the three vectors of the raw data in (D) a non-dyskinetic control and (E) a dyskinetic patient. 
Table 2 Mean clinical rating scores of all dyskinetic patients and mean clinical rating scores of dyskinetic patients falling within the task specific normal range of acceleration

\begin{tabular}{|c|c|c|c|c|c|c|}
\hline Task No & Description & $\begin{array}{l}\% \text { Dyskinetic patients } \\
\text { within normal ranges } \\
(\text { actual No })^{\star}\end{array}$ & $\begin{array}{l}\text { Mean AIMS score of } \\
\text { dyskinetic patients } \\
\text { within normal range }\end{array}$ & $\begin{array}{l}\text { Mean AIMS score of } \\
\text { all dyskinetic patients } \\
\text { (range) }\end{array}$ & $\begin{array}{l}\text { Mean Goetz score } \\
\text { of patients within } \\
\text { normal ranges }\end{array}$ & $\begin{array}{l}\text { Mean Goetz score of } \\
\text { all dyskinetic patients } \\
\text { (range) }\end{array}$ \\
\hline 1 & Sit, silent & $11(1 / 9)$ & 0.5 & $8.88(0.5-19.5)$ & 1 & $1.7(0.5-3)$ \\
\hline 2 & Mental taxation & $11(1 / 9)$ & 4 & $11.63(3.5-21.5)$ & 1 & $1.87(1-4)$ \\
\hline 3 & Conversation & $11(1 / 9)$ & 0.5 & $11.63(4-16.5)$ & 1 & $1.79(1-3)$ \\
\hline 1,2 , and 3 & "Sitting tasks" & $11(1 / 9)$ & 2.67 & $10.86(0.5-21.5)$ & 1 & $1.69(0.5-4)$ \\
\hline 4 & Writing & $12.5(1 / 8)$ & 2 & $9.875(1.5-17)$ & 1 & $1.68(1-2.5)$ \\
\hline 5 & Drinking & $12.5(1 / 8)$ & 3.5 & $9.41(3-15.5)$ & 1 & $1.38(1-2.5)$ \\
\hline 6 & Preparing toast & 0 & - & $10.66(3-19)$ & - & $1.83(1-3)$ \\
\hline 7 & Eating toast & 0 & - & $11.17(4.5-21.5)$ & - & $1.75(1-3)$ \\
\hline 6 and 7 & (combined) & 0 & - & $10.9(3-21.5)$ & - & $1.75(1-3)$ \\
\hline 8 & Coat & $37.5(3 / 8)$ & 6.6 & $8.25(1-19)$ & 1 & $1.72(1-3.5)$ \\
\hline $1-8$ & All tasks - walking & $11(1 / 9)$ & 4.8 & $18.76(0.1-19.1)$ & 1 & $1.51(0.1-1.53)$ \\
\hline 9 & Walking & $100(12 / 12)$ & $7.5(0.5-15.5)$ & $7.5(0.5-15.5)$ & 1.54 & $1.54(1-2)$ \\
\hline
\end{tabular}

^Not all patients exhibited dyskinesias in all tasks.

PRELIMINARY RECORDINGS

A total of 26 subjects (16 patients with Parkinson's disease and levodopa induced dyskinesias and 10 controls), recruited through the outpatient department, were studied. Six subjects (four patients and two controls) were used in the preliminary recordings and 20 subjects in the validation of the device against established clinical rating scales. In both groups, the triaxial accelerometer was taped to the shoulder (of the worst affected side in the patients, and the dominant side in controls) posteromedial to the acromioclavicular joint. This site was chosen after pilot recordings demonstrated that accelerometers placed here were sensitive to both axial and proximal upper limb movements and, even to leg movements (such as in walking) transmitted to the trunk. Patients and controls were asked to perform various tasks, such as talking, writing, and walking, while wearing the ambulatory monitor. The effect of these timed activities was then observed on both the raw data trace and the acceleration in different frequency bands.

VALIDATION OF DATA AGAINST ESTABLISHED CLINICAL RATING SCALES

Twelve patients with LID and eight age matched controls followed a set protocol to enable correlation with the clinical rating scales. In the patient group, there were eight men and four women, mean age 55.75 (range 42-74) years, and in the control group, five men and three women, mean age 62.75 (50-73) years. Mean duration of disease was 14.6 (10-20) years, mean duration of levodopa therapy was $12(8-16)$ years, and mean Hoehn and Yahr stage (off) was 4 (3-5).

Subjects were monitored over a period of 20 minutes. During this time they were video recorded at rest, and during mental activation and various motor tasks (table 1 ).

The activities were chosen to include those in the Goetz scale (5, 8, and 9) plus a further four activities which we thought particularly likely to provoke dyskinesias or represent everyday activity. Not all patients were capable of mental arithmetic, in which case mental taxation was performed by asking questions from the mini mental state examination. ${ }^{12}$ During the recording, nine of the patients remained on with dyskinesias of varying severity, and three patients were off.
The videos were then analysed by applying modified Goetz and AIMS scales to each of the eight tasks (while retaining the original scoring systems of each scale). The Goetz scale was modified firstly by excluding distinctions between chorea, dystonia, or other forms of dyskinesia, and secondly, by including the extra tasks. The five point disability scale was applied to the motor tasks (4-9) directly, and to the ability to sit still on the chair for tasks $1-3$. The AIMS scale was modified, by exclusion of orofacial and mandibular dyskinesia grading, by observing the patients during the aforementioned activation, and also by omitting the subjective patient rating section. Each patient's video recording was assessed independently by two neurologists (AJM and JO'S), before the analyses of the accelerometry data.

As preliminary recordings demonstrated dyskinesias to principally lie in the $1-3 \mathrm{~Hz}$ frequency band, the acceleration profiles were then measured for each subject in the $1-3 \mathrm{~Hz}$ frequency bands using the customised soft-
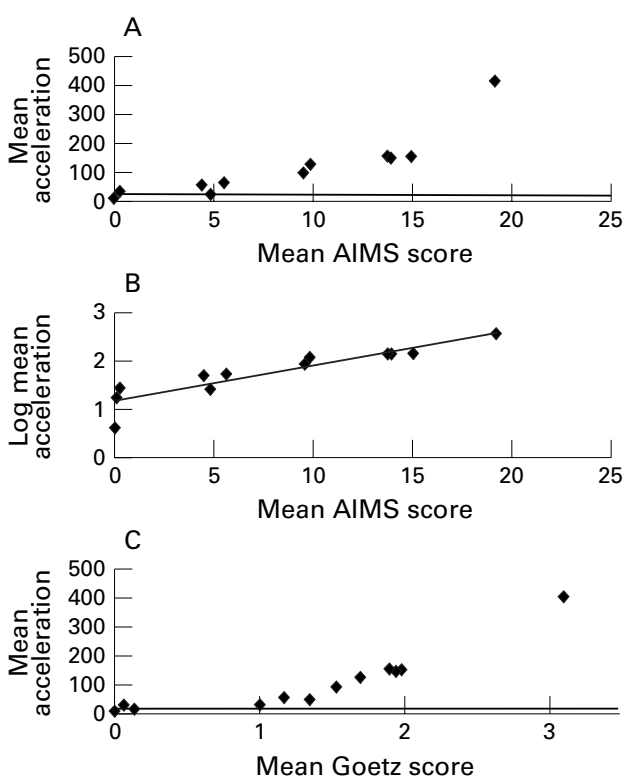

Figure 2 Mean accelerations in the $1-3 \mathrm{~Hz}$ frequency band (in arbitrary units) per patient plotted against mean clinical rating scores per patient for the whole recording period (excluding walking). (A) Mean accelerations $v$ mean AIMS scores. (B) Logged mean accelerations v mean AIMS scores. (C) Mean accelerations $v$ mean Goetz scores. Solid lines ( $A$ and $C$ ) represent upper limit of "normal" ranges. 

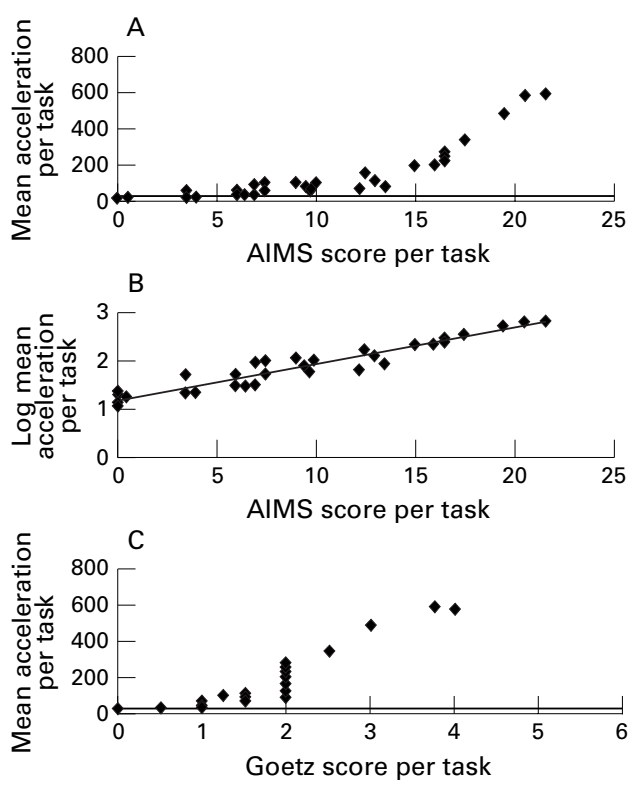

Figure 3 Mean accelerations in the 1-3 Hz frequency band (in arbitrary units) per sitting task plotted against mean clinical rating scores per task. (A) Mean accelerations $v$ mean AIMS scores. (B) Logged mean accelerations $v$ mean AIMS scores. (C): Mean accelerations $v$ mean Goetz scores. Solid lines ( $A$ and $C$ ) represent upper limit of "normal" ranges.

ware. Mean acceleration per second for each task was correlated against mean clinical dyskinesia rating for each patient in each task. As preliminary recordings demonstrated that walking produced high accelerations, even in controls, periods of walking were excluded from the final analysis. Thus, mean acceleration per second for the whole period, excluding walking, was then correlated against mean dyskinesia ratings for all tasks except walking. The control means $\pm 2 \mathrm{SD}$ were used to estimate normal range for acceleration for the tasks.

\section{STATISTICAL ANALYSIS}

Spearman's rank test was used to correlate accelerations per task against both clinical rating scales for all tasks. Both scales were analysed individually. The three "sitting tasks" (tasks 1, 2, and 3) were analysed together, as were preparing and eating toast (tasks 6 and 7). Mean accelerations for the whole recording time (except time spent walking) were then

Table 3 Correlations of the various activation tasks and combined results with the AIMS and Goetz scales

\begin{tabular}{|c|c|c|c|c|}
\hline \multirow[b]{2}{*}{$\begin{array}{l}\text { Task } \\
\text { No }\end{array}$} & \multirow[b]{2}{*}{ Description } & \multicolumn{2}{|c|}{ Acceleration vs AIMS score } & \multirow{2}{*}{$\begin{array}{l}\text { Acceleration v Goetz } \\
\text { Spearman rank } \\
\text { correlation }\end{array}$} \\
\hline & & $\begin{array}{l}\text { Spearman rank } \\
\text { correlation }\end{array}$ & $\begin{array}{l}\text { Pearson's logged } \\
\text { acceleration }\end{array}$ & \\
\hline 1 & Sit silent & $0.95^{\star \star \star}$ & & $0.94^{\star \star \star}$ \\
\hline 2 & Mental activation & $0.99^{\star \star \star}$ & & $0.98^{\star \star \star}$ \\
\hline 3 & Conversation & $0.97^{\star \star \star}$ & & $0.94^{\star \star \star}$ \\
\hline 1,2 , and 3 & "Sitting tasks" & $0.98^{\star \star \star}$ & $0.96^{\star \star \star}$ & $0.96^{\star \star \star}$ \\
\hline 4 & Write & $0.97^{\star \star \star}$ & $0.91^{\star}$ & $0.95^{\star \star \star}$ \\
\hline 5 & Drink & $0.88^{\star \star \star}$ & $0.95^{\star}$ & $0.78^{\star}$ \\
\hline 6 & Prepare toast & $0.92^{\star \star \star}$ & $0.96^{\star \star \star}$ & $0.87^{\star \star \star}$ \\
\hline 7 & Eat toast & $0.93^{\star \star \star}$ & & $0.87^{\star \star}$ \\
\hline $6 \& 7$ & $\begin{array}{l}\text { Prepare and eat } \\
\text { toast }\end{array}$ & $0.97^{\star \star \star}$ & & $0.85^{\star \star \star}$ \\
\hline 8 & Coat & $0.82^{\star \star}$ & 0.87 & 0.77 \\
\hline $1-8$ & All tasks & $0.97^{\star \star \star \star}$ & $0.91^{\star \star \star}$ & $0.95^{\star \star \star}$ \\
\hline 9 & Walk & $0.72^{\star}$ & $0.63^{\star \star}$ & 0.63 \\
\hline
\end{tabular}

(All p values $\left.<0.05 ;{ }^{\star} \mathrm{p}<0.01 ;{ }^{\star \star} \mathrm{p}<0.02 ;{ }^{\star \star \star} \mathrm{p}<0.001\right)$ correlated with mean clinical rating scores (excluding walking). These were correlated using Spearman's rank test for both scales. As preliminary recordings had suggested an exponential trend in the correlation, to demonstrate this, Pearson's test was used to correlate logged accelerations with AIMS scores. The scores of each rater were correlated ( using Pearson's test for the AIM scale, and Spearman's rank test for the Goetz scale) and the mean of the two values was taken as the clinical rating score for each task.

\section{Results}

PRELIMINARY RECORDINGS

Dyskinetic movements were principally represented in the 1-3 Hz frequency bands. The acceleration caused by voluntary movements in normal people (except for walking), (fig $1 \mathrm{~A}$ ) was much less than the acceleration resulting from dyskinetic activity (fig $1 \mathrm{~B}$ ). Periods of tremor were represented by a peak at $4-6 \mathrm{~Hz}$ and could be easily recognised on the raw data trace, (fig $1 \mathrm{C}$ ).

Walking produced high accelerations in both normal controls and dyskinetic and nondyskinetic (off) patients and had a characteristic pattern for both patients and controls, on the raw data trace, most marked in the vertical plane (fig $1 \mathrm{D}$ and $\mathrm{E}$ ).

VALIDATION AGAINST CLINICAL RATING SCALES There was overlap into the estimated normal range by accelerations produced by very mildly dyskinetic patients only (table 2).

As the patient and control groups were only moderately well matched for age, with a small but not significant difference, we performed Pearson's tests on accelerations against age for both groups. No correlation was found between age and acceleration values in either the controls or the patients, so we concluded that this difference was unlikely to influence the results.

Acceleration per second correlated well against both clinical scales for all tasks, with what seemed to be an exponential trend. The correlations were statistically significant.

Mean acceleration per patient during the whole recording time (except the section spent walking), correlated very strongly against the modified AIMS scale per patient $(r=0.97$, $\mathrm{p}<0.001$, fig $2 \mathrm{~A}$ ) and against the modified Goetz scale per patient $(r=0.95$, p $<0.001$, fig 2 C). The exponential trend is demonstrated by a strong correlation $(r=0.91, p<0.001)$ between the logged accelerations per patient and the mean AIMS scales per patient, (fig $2 \mathrm{~B}$ ).

Mean acceleration during all sitting tasks also correlated very strongly against the modified AIMS ( $r=0.98, \mathrm{p}<0.001$, fig $3 \mathrm{~A})$ and Goetz ( $\mathrm{r}=0.96, \mathrm{p}<0.001$, fig $3 \mathrm{C}$ ) scales per task. Logged accelerations correlated strongly with mean AIMS scores $(r=0.96, \mathrm{p}<0.0001$, fig $3 \mathrm{~B})$.

Walking correlated least well and there was a large overlap with the $95 \%$ confidence interval ( $95 \%$ CI) for the normal range, (table 2$)$. The rest of the results are summarised in table 3 . 
Correlations between the two raters' scores were high for the individual tasks and for the combined scores for both the AIMS (mean $r=0.95$, range 0.89-0.97, all $\mathrm{p}<0.05$ ) and the Goetz scale ( mean $r=0.9$, range $0.79-0.97$, all $\mathrm{p}<0.01$ )

Different topographical sites were affected equally by dyskinesia, with mean AIMS scores (all patients) for neck, trunk, upper limbs, and lower limbs of $1.75,1.74,1.74$, and 1.76, respectively.

Only one patient (who weighed less than 45 $\mathrm{kg}$ ) complained that she found the device cumbersome.

\section{Discussion}

The results obtained with the ambulatory monitor correlate extremely well with currently available research measures of dyskinesia. Although the scales have been modified, it is unlikely that their validity is less as each correlates well and there is good interrater reliability. Furthermore, neither is commonly used as originally validated. As the Goetz scale relies more on disability caused by dyskinesias, and the AIMS scale more on dyskinesia severity, it is not surprising that the latter correlates somewhat better with our method, which is not designed to assess disability.

Overall mean accelerations for the whole recording period correlated well with mean clinical ratings for all tasks (walking excluded) and for both scales. The data thus support the value of repeated "snapshot" clinical rating scale assessments as a representative measure of overall dyskinesia severity. The fact that these correlations were maintained during writing and eating, with little overlap into the normal range, suggests that the device has specificity for dyskinesia measurement, even during voluntary movement.

Except for walking, correlations for putting on a coat were weakest. This may be partly due to the vigorous nature of the activity and partly due to displacement of the accelerometer during the task. However, this type of activity is infrequent in normal daily activities.

Previous studies have demonstrated the ability to measure tremor and periods of hypokinesia using similar techniques. ${ }^{8-10}{ }^{13}$ Hoff et al used a similar multidirectional accelerometer based technique to measure dyskinesias and found good correlation with clinical rating scales in the absence of voluntary movement. ${ }^{14}$ However, they used several pairs of biaxial accelerometers, mounted at different sites on the body. Our choice of location for the triaxial accelerometer proved to be sensitive for dyskinesias, and all body parts were equally represented by dyskinesias in clinical rating. In general it is unusual to see significant limb dyskinesias without coexistent marked axial dyskinesias, ${ }^{14}$ although the reverse can occur.

One of the difficulties in developing ambulatory devices in the past has been that voluntary movement tends to occur at similar frequencies to those dominated by dyskinesia. ${ }^{13}$ By studying non-dyskinetic people, we have established a normal range of acceleration for certain, typical activities of daily living, and found dyski- netic activity generally to be well in excess of the normal range. Accelerations above the normal range clearly relate to severity of dyskinesia. By producing an acceleration trace, which is a much more descriptive means of recording movement than simple accelerometry counts, our device enables easy recognition of relevant activities, such as walking, and tremor, which both have characteristic features on the raw data trace. Use of the original data trace and choice of frequency band analysis (which excludes parkinsonian tremor) ${ }^{15}$ has enabled us to develop a much more specific method of dyskinesia measurement than accelerometer based devices in the past.

An obvious advantage of this method of dyskinesia assessment is the relatively small demand on clinical time. The computer programme provides a fast, efficient, and accurate means of measuring the relevant acceleration, which is less subject to bias and human error. Furthermore, it does not require a trained neurologist to gather the data, which could be collected by a technician or nurse specialist. It also avoids the need to analyse interrater reliability, and rigorous blinding strategies, in the context of clinical trials.

The main limitation of this preliminary study is the low specificity for very mild dyskinesias (with some overlap into the estimated normal range). However, we envisage that the demand would mostly be for measurement of more moderate and severe dyskinesias. Another drawback is the need to exclude walking from the analysis. However, patients complaining of intolerable dyskinesias only occasionally complain of the effect on walking, and seem generally to be troubled more during more sedentary activities. This method may still prove useful for measuring intrapatient differences in walking and milder dyskinesias, although validation may be more difficult. This device is also inappropriate for orofacial dyskinesias, dystonias, and stereotypies, which can appear at onset and end of dose. However, the pathophysiological difference between these and the predominantly choreiform peak dose movements is unclear, ${ }^{14}$ as is the benefit of differentiating between them in phase 2 trials. In this study, we did not differentiate between dystonic, choreiform, or other types of dyskinesias in the clinical rating but there was still good correlation with the acceleration profiles.

Another criticism could be of the chosen site and mounting technique for the accelerometer. However, we have found that patients rarely display significant limb dyskinesias without axial involvement, and as patients' individual dyskinesia phenomenology tends not to change with time, this would not be an issue when comparing intrapatient changes, for which this device is ultimately designed. Also, in our study different topographical areas were equally affected by dyskinesia, and yet correlations were still high. In principle, accelerometers taped to the skin will not necessarily record whole body motion because skin may move independently or attenuate the transmission of higher frequencies. In practice, these potential limitations did not 
seem important over the $1-3 \mathrm{~Hz}$ frequency band primarily used in this study.

Our study has been performed in a highly controlled situation, and the assumptions made have yet to be confirmed in unsupervised ambulatory conditions, over longer periods. These studies are now in progress. The ability to demonstrate off period hypokinesia, using a similar accelerometry based technique, has previously been demonstrated, ${ }^{9} 16$ and, in the ambulatory setting, it will be necessary to exclude these periods as well as hyperkinetic periods of walking. We also plan to study larger numbers of age matched controls and nondyskinetic patients with Parkinson's disease, to confidently establish a valid normal range.

Although our device is portable and quite compact, we hope to develop it further, making it lighter and smaller. It may also be useful to vary the site of the accelerometer while assessing validity with different types of dyskinesia.

There is no gold standard clinical rating scale and those used are often modified from their original format, thus potentially compromising their validity. ${ }^{17}$ A practical reliable method of objective dyskinesia measurement is needed. We think that our device accurately measures dyskinesias. We are hopeful that this method will soon provide a vital part of assessment in phase 2 clinical trials, and in the clinical evaluation of levodopa induced dyskinesias.

We are grateful to the Virginia Keiley Benefaction for providing funding for purchase of the equipment. We thank Dr Jeremy Hobart and Jenny Head for helpful discussion and statistical advice.

1 Brotchie J. Adjuncts to dopamine replacement: a pragmatic approach to dealing with the problem of Dyskinesia in Parkinson's disease. Mov Disord 1998;13:871-6.
2 Quinn N, Bhatia K. Functional neurosurgery for Parkinson's disease has come a long way, though much remains experimental. BMF 1998;316:1259-60.

3 Nutt JG. Levodopa-induced dyskinesia: review, observations, and speculations. Neurology 1990;40:340-5.

4 May PRA, Lee MA, Bacon RC. Quantitative assessment of neuroleptic-induced extra-pyramidal symptoms; clinical and non-clinical approaches. Clin Neuropharmacol 1983; 6(suppl 1):35-51.

5 Goetz CG, et al. Utility of an objective dyskinesia rating scale for Parkinson's disease: inter- and intrarater reliability assessment. Mov Disord 1994;9:390-4

6 Goetz CG, Stebbins GT, Blausucci LM, et al. Efficacy of a patient-training videotape on motor fluctuations for on-off diaries in Parkinson's disease. Mov Disord 1997;12:103941.

7 Golbe LI, Pae J. Validity of a mailed epidemiological questionnaire and physical self-assessment in Parkinson's disease. Mov Disord 1998;3:245-54.

8 Van Hilten JJ, Middelkoop HAM, Kerkhof GA, et al. A new approach in the assessment of motor activity in Parkinson's disease. F Neurol Neurosurg Psychiatry 1991;54:976-9.

9 Van Hilten JJ, Kabeil JF, Middelkoop HAM, et al. Assessment of response fluctuations in Parkinson's disease by ambulatory wrist activity monitoring. Acta Neurol Scand 1993;87:171-7

10 Hoff JI, van der Plas A, Wagermans EAH, et al. Objective quantification of levodopa-induced dyskinesias in Parkinson's disease using multi-channel accelerometry [abstract]. Mov Disord 1998;13 (suppl 2):222.

11 Manson AJ, Brown P, Bedlington R, et al. An ambulatory device for recording dyskinesias [abstract]. Mov Disord 1998;13(suppl 2):170.

12 Folstein MF, Folstein SE, McHugh PR. Mini-mental state. A practical method for grading the cognitive state of patients for the clinician. F Psychiatr Res 1975;12:189-98.

13 Redmond DP, Hegge FW. Observations on the design and specification of a wrist-worn human activity monitoring
system. Behaviour Research Methods, Instruments, and system. Behaviour Resear
Computer 1985:659-69.

14 Marconi R, Lefebre-Caparros D, Bonnet AM et al. Levodopa-induced dyskinesias in Parkinson's disease: phenomenology and pathophysiology. Mov Disord 1994:9:212 .

15 Dubinsky RM. Some physiological aspects of Parkinsonian tremor. In: Findley LF, Koller WC, eds. Handbook of tremor disorders. New York: Marcel Dekker, 1995:313-18.

16 Dunnewold RJW, et al. Ambulatory quatitative assessment of body position, bradykinesia, and hypokinesia in Parkinson's disease. F Clin Neurophysiol 1998;15:235-42.

17 Hobart JC, Lamping DL, Thompson AJ. Evaluating neurological outcome measures: the bare essentials. F Neurol Neurosurg Psychiatry 1996;60:127-30. 